\title{
A Review of Primary Mine Ventilation System Optimization
}

\author{
Enrique I. Acuña \\ Facultad de Ingeniería y Ciencias Aplicadas, Universidad de los Andes, Santiago, Chile, \\ Proyecto Nuevo Nivel Mina, Vicepresidencia de Proyectos, CODELCO, Chile, enacuna@ gmail.com \\ Ian S. Lowndes \\ Process and Environmental Research Division, Faculty of Engineering, University of Nottingham, Nottingham, United Kingdom, \\ ian.lowndes@nottingham.ac.uk
}

Within the mining industry, a safe and economical mine ventilation system is an essential component of all underground mines. In recent years, research scientists and engineers have explored operations research methods to assist in the design and safe operation of primary mine ventilation systems. The main objective of these studies is to develop algorithms to identify the primary mine ventilation systems that minimize the fan power costs, including their working performance. The principal task is to identify the number, location, and duty of fans and regulators for installation within a defined ventilation network to distribute the required fresh airflow at minimum cost. The successful implementation of these methods may produce a computational design tool to aid mine planning and ventilation engineers. This paper presents a review of the results of a series of recent research studies that have explored the use of mathematical methods to determine the optimum design of primary mine ventilation systems relative to fan power costs.

Key words: mine ventilation optimization; linear programming; nonlinear programming.

History: This paper has been refereed.

The design of reliable mine ventilation systems is key to the safe and economical operation of underground mines. Governments of many countries recognize the important role of ventilation in the safety of underground mines by imposing national mining laws and regulations. These 
rules specify the minimum safety and environmental conditions that a ventilation system must maintain within a working underground mine. The main objectives of such a system are to sustain life and to ensure a safe and acceptable working environment. Achieving these aims requires the delivery of a sufficient quantity of fresh air to ensure the rapid dilution of mine pollutants to below statutory occupational exposure levels (OELs). These pollutants may include strata gases (e.g., methane and radon), mineral dust and fumes, and particulates from dieselpowered equipment.

Mine ventilation engineers currently use personal experience and good practice guidelines to identify the best mine ventilation arrangements by the repeated use of steady state mine ventilation network programs to evaluate the airflow and pressure distribution. However, this method is time consuming and may not always identify the solution that delivers the minimum fan power cost. The challenge for the engineer is to identify a practical subset of ventilation arrangements and performance levels that deliver the desired airflow distribution. The use of any new ventilation arrangement needs to satisfy all mining, health, and safety laws of the country in which the mine operates. For example, in the United States, any significant changes to a mine ventilation system need the prior approval of the Mine Safety and Health Administration. National mining laws can differ between countries. For example, the European Union allows booster fans in coal mines, whereas the United States prohibits them.

The principal optimization problem associated with underground mine ventilation systems is to determine the number, location, and duty of the fans and regulators to deliver the needed airflow and pressure distribution at the lowest fan power or energy consumption. 
This paper provides (1) a definition of the objectives that govern the design and operation of a main mine ventilation system, (2) a summary of the interconnected tunnels that form the mine network, and (3) a review of the engineering components (including fans and regulators) that distribute the fresh air within the mine network. We present a mathematical formulation of the objective function and constraints that define the optimization problem, and follow this with a review of the optimization methods proposed to find the best engineering solution. The optimization problem seeks to minimize the ventilation power consumption of a defined network, subject to the conservation of mass flow and energy, the satisfaction of upper and lower airflow bounds, and any controls on the location of fans and regulators (Barnes 1989). Large mine networks can be complex; they often comprise hundreds of interconnected roadways, which are ventilated by fans and regulators positioned around the network. Minimizing the power costs of these systems is a challenging, practical problem that may be examined as a solution to a highly nonlinear mathematical problem.

\section{Primary Mine Ventilation Systems}

The layout of interconnected tunnels formed to access and exploit the underground mineral deposits defines the topology of an underground mine ventilation network. The design of a ventilation system is determined principally by the location and rates of generation of the contaminants within the mine circuit. Most pollutant emissions occur at the working faces. The working face is that portion of a tunnel in which minerals are actively extracted. Although the design of each individual mine ventilation system is unique, each system has similar infrastructural and operational characteristics. Vertical shafts or inclined tunnels, commonly termed ramps, connect the underground network of tunnels to the surface. These surface 
connections serve as (1) egress routes for workers, mobile machinery, and materials, (2) conveyance paths for the extracted mineral, and (34) entry or exit portals for the ventilation air.

Figure 1 shows a generic layout of a mine ventilation network, including typical locations of doors and airflow distribution controls represented by regulators and fans. Separating the flow paths provided by the tunnels that form a mine network into a fresh (intake) and a contaminated (return) flow circuit is normal. Roadways fitted with airlock doors interconnect these two flow circuits. The double D (DD) symbol in Figure 1 represents airlock doors. These doors prevent the short circuit of the fresh air to the contaminated return air circuit, and allow for the safe and efficient movement of workers, equipment, and supplies between the two airflow circuits.

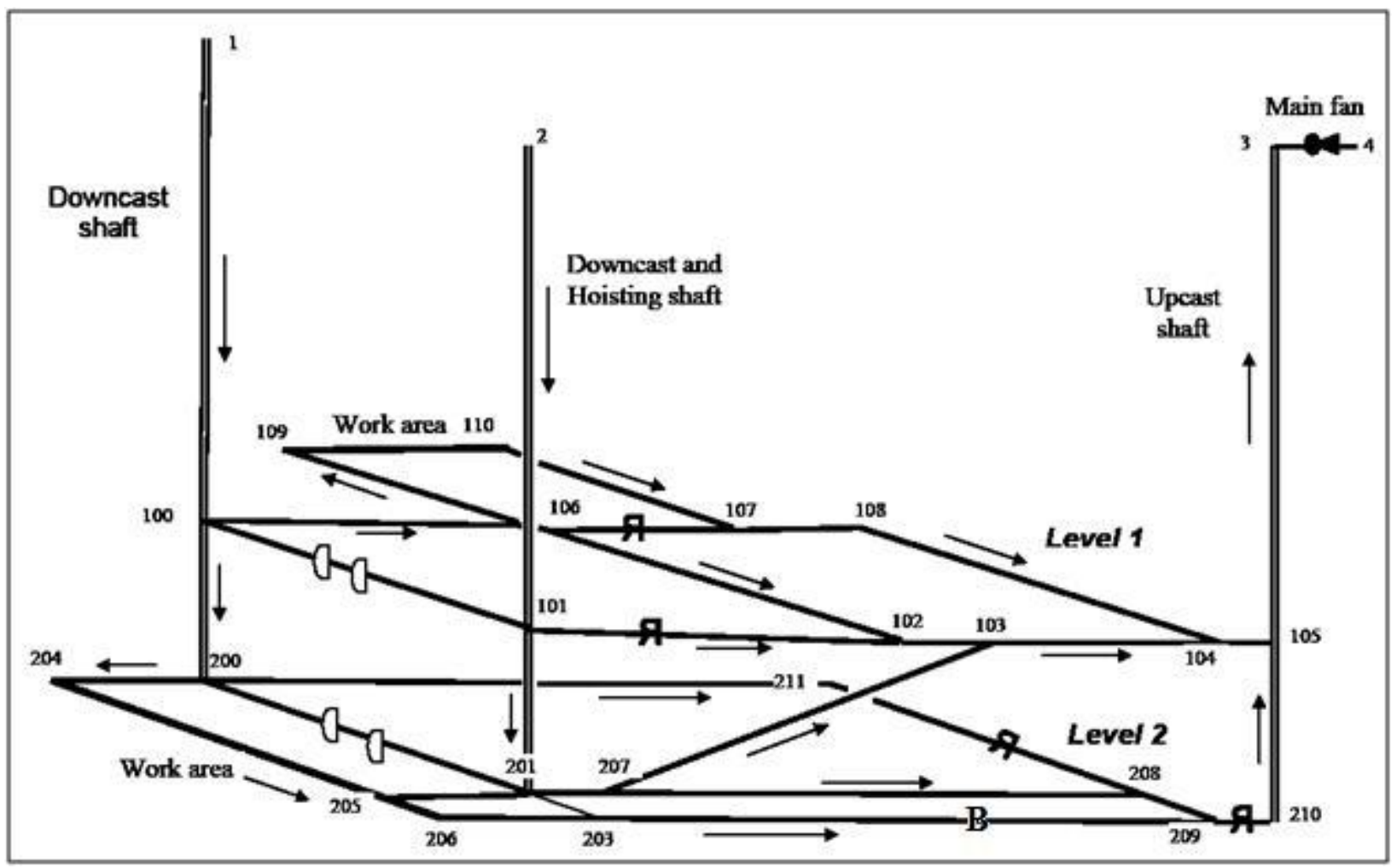


Figure 1: This example of a generic mine ventilation network shows the direction of airflow and typical locations of the ventilation devices, such as doors (DD), main fan, booster fan (B), and regulators $(\mathrm{R})$, which maintain the required airflow distribution.

Source: McPherson (1993).

To create a controlled flow of fresh air to all working areas, engineers supplement any natural thermal airflow drafts with large main fans, normally located at or near the surface. These main fans either push fresh air into, or pull contaminated air out of, the underground mine ventilation network. Booster fans and air regulator doors divide the fresh airflow entering the mine between the various mine workings. The $\mathrm{B}$ and $\mathrm{R}$ symbols in Figure 1 represent the locations of a booster fan and airflow regulators, respectively. The regulators throttle the airflow passing through a particular airway and distribute the fresh airflow delivered by the main fan.

Booster fans are added when the main fan(s) cannot deliver the pressure to transport the needed airflow quantities to the furthest locations in the mine. This may occur when a mine ventilation network expands to exploit mineral reserves at an increased distance or depth from the surface connections. Where local mining laws and regulations allow, underground booster fans may be installed within selected branches to deliver the energy to overcome the pressure losses within the circuit and preserve the required ventilation flow rates. The correct design and installation of booster fans should minimize uncontrolled recirculation, but not adversely influence the performance of other fans installed in the network.

Within a mine, there are working locations in which the pollutant necessitates a minimum airflow quantity to dilute it below statutory OELs. These working locations are termed specified airflow branches. The mining activities that produce pollutants include mineral production faces, diesel maintenance workshops, battery and fuel charging stations, and mineral crusher stations. 


\section{Mathematical Representation}

A directed graph composed of nodes and edges, termed junctions and branches by ventilation practitioners (Moll and Lowndes 1992), mathematically represents the topography of a primary mine ventilation network (PMVN). The edges represent the different paths airflow can follow and the nodes are the connections between those paths. Mine ventilation network calculations employ the defined network topography, apply Kirchhoff's first and second circuit laws, and ensure that the pressure drops and airflows in each branch satisfy the Atkinson equation. These laws ensure that the calculated airflow distribution complies with the principles of mass and energy conservation (McPherson 1993, Lowndes and Tuck 1995). Equations 2 to 15 in the appendix mathematically model these physical principles.

Kirchhoff's first law states the mass flow entering a junction (node) is equal to the mass flow leaving that junction. Equation (15) represents the balance of the mass flow entering and leaving the $i^{\text {th }}$ junction of a network of connected branches, where $a_{i j}=1$ if the $j^{\text {th }}$ branch connects to the $i^{\text {th }}$ junction and the air flows out of the junction; $a_{i j}=-1$ if the $j^{\text {th }}$ branch connects to the $i^{\text {th }}$ junction and the air flows into the $i^{\text {th }}$ junction; and $a_{i j}=0$ if the $j^{\text {th }}$ branch does not connect to the $i^{\text {th }}$ junction. For many mine ventilation networks, the changes in air density at a given junction are small, which simplifies Equation (15) to Equation (2). This new equation states that the volumetric airflows entering a junction (node) are equal to the volumetric airflows leaving that junction. When a flow is compressible, we consider the changes in density; see Equation (15). If the flow is incompressible, density does not change or changes in density are not relevant and Equation (2) is applicable. Usually, as McPherson (1993) indicates for shallow mines with depths of 500 meters or less, density does not change significantly. 
Kirchhoff's second law states that the sum of the directed energy differences (i.e., potentials and losses) around a closed mesh is zero. In a ventilation network, the energy potentials are the pressure energies delivered by the fans and the thermal drafts. The frictional resistance and the shock losses create the energy losses experienced within the airways or roadway. Changes in the roadway cross section, flow around a bend in the roadway, and flow around equipment within the roadway generate changes to the flow direction or speed. The changes in the flow direction or speed result in energy losses, termed shock losses. The shock pressure losses, caused by changes in the flow direction or speed within the $j^{\text {th }}$ branch, are often estimated as the frictional pressure drop created by an equivalent length of the host branch. This equivalent length $\left(s_{j}^{\text {equiv }}\right.$ ) is then added to the actual physical length of the host airway to calculate the total frictional and shock pressure drop experienced within this branch.

For incompressible and turbulent flow, Equation (3) represents the frictional and shock pressure losses $\left(L_{j}\right)$ experienced within the $j^{\text {th }}$ branch, which is proportional to the square of the volumetric flow rate $\left(Q_{j}\right)$ in that branch. The constant of proportionality, termed the total resistance of branch $\left(r_{j}\right)$, is determined from the evaluation of Atkinson's equation (McPherson 1993), as Equation (4) represents. The total resistance $\left(r_{j}\right)$ is equal to the product of the friction factor $\left(k_{j}\right)$, the perimeter $\left(p_{j}\right)$, and the sum of the length and equivalent shock loss length $\left(s_{j}+\right.$ $\left.s_{j}^{\text {equiv }}\right)$ of the branch, divided by the cube of the cross-sectional area $\left(A_{j}\right)$ of the branch.

Equation (5) represents the shock pressure loss $\left(R_{j}\right)$ because of a regulator within the $j^{\text {th }}$ branch. We can also estimate $R_{j}$ as the frictional pressure drop created by an equivalent length $\left(s_{j}^{r e g}\right)$ of the host branch. We determine the resistance generated by the regulator $\left(r_{j}^{*}\right)$ in the host airway from the evaluation of the Atkinson's equation; see Equation (6). 
When heat is transferred to the air, a thermal draft, termed the natural ventilation pressure, produces pressure differences within a mine network. High temperature sources that produce a flow of heat to the air include the surrounding rock and installed equipment. Thus, for an incompressible and turbulent airflow, the total pressure drop $\left(H_{j}\right)$, experienced within the $j^{\text {th }}$ branch of a mine network, is determined by the sum of four pressure drop components: the frictional pressure loss $\left(L_{j}\right)$, the shock pressure loss of a regulator $\left(R_{j}\right)$, the fan pressure gain $\left(F_{j}\right)$, and the natural ventilation pressure gain $\left(N_{j}\right)$. Equation (7) represents the total pressure drop in the $j^{\text {th }}$ branch. Kirchhoff's second circuit law states that the algebraic sum of all the total pressure drops experienced around the $i^{\text {th }}$ closed path in the network (termed the $i^{\text {th }}$ fundamental mesh $M_{i}$ ) is equal to zero. Equation (8) represents this energy balance, as Figure 2 represents graphically.

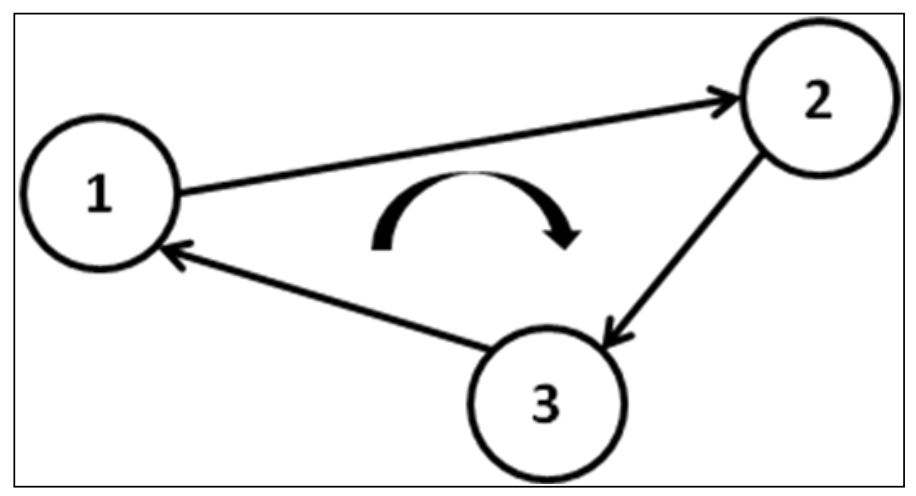

Figure 2: This example of a closed path or fundamental mesh comprises three nodes and

\section{three branches in a network.}

The arrow alongside each branch in Figure 2 shows the direction of airflow. The curved arrow at the center of the mesh indicates the chosen direction of the flow in the mesh. If $\mathrm{Nb}$ is the total number of branches and $\mathrm{Nn}$ is the total number of nodes in the ventilation network, then $\mathrm{Nm}$ $(N m=N b-N n+1)$ defines the number of fundamental meshes (closed paths) present in the network. In Equation (8), $b_{i j}$ is the coefficient that multiplies the pressure drop on each branch $\left(H_{j}\right) ; b_{i j}=1$ if the $i^{t h}$ mesh contains the $j^{t h}$ branch and the flow within this branch is in the same 
direction as that defined for the mesh; $b_{i j}=-1$ if the $i^{\text {th }}$ mesh contains the $j^{\text {th }}$ branch and the flow within this branch is in the opposite direction as that defined for the mesh; and $b_{i j}=0$ if the $i^{t h}$ mesh does not contain the $j^{\text {th }}$ branch.

For a given mine layout, the engineers determine the location and airflow requirements of each working zone within the mine. The rates of mineral production and transport define the levels of pollutants created in the mine. This knowledge allows the ventilation engineer to determine the minimum and maximum airflow quantities delivered by the ventilation system (McPherson 1993). Equations (9) and (10) mathematically represent these constraints, where the range of the air quantity flowing through the $j^{\text {th }}$ branch $\left(Q_{j}\right)$ is restricted by a lower $\left(l_{j}\right)$ and an upper $\left(u_{j}\right)$ bound.

Additionally, Equations (11) and (12) represent the fan air power and energy consumption, respectively. $P_{j}$ is the delivered air power and $\eta_{j}$ is the total efficiency of the fan located in the $j^{\text {th }}$ branch of the network. However, practical constraints may preclude the installation of regulators or fans within certain mine roadways. For example, booster fans are not normally installed within major transport or mineral conveyance roadways. To exclude a regulator or booster fan within the $j^{\text {th }}$ branch, the regulator pressure loss term $\left(R_{j}\right)$ or the fan pressure term $\left(F_{j}\right)$ is zero.

For a given set of initial conditions, the linear and nonlinear equations defined by Equations (2) to (8) are solved, using commercially available programs, to evaluate the steady state distribution of airflows and pressures within a given mine ventilation network. The most popular iterative method used to perform this steady state evaluation of a mine ventilation network airflows and pressures is the Hardy Cross method (Cross 1936). The Hardy Cross method is an example of a single-step Newton-Raphson numerical scheme. The algorithm first 
identifies an initial feasible solution for the balanced flows within the network. The method then iteratively determines the corrections needed to balance the calculated pressure losses around each mesh in the network and calculates the changes needed to the flow estimates. The process repeats until consecutive solutions satisfy a chosen convergence criterion. For specified airflow branches, the method determines the value of the equivalent resistance or fan pressure added to this branch to achieve the required minimum airflow quantity.

Several commercial mine ventilation network programs are available to evaluate the steady state volumetric flow and pressure distribution within a mine ventilation network. The evaluation of the steady state airflow and pressure distribution can be manually calculated using the Hardy Cross method, but it is a tedious and time-consuming process. Table 1 summarizes examples of these programs (Hardcastle 1995, Wallace 2001, Widzyk-Capehart and Watson 2001, Marx and Belle 2002). Most of these programs use an accelerated form of the Hardy Cross iterative solution method (McPherson 1993). The computational algorithms and user interfaces of these software tools are similar. These programs employ a graphical user interface to enter and display (1) the branch properties, (2) the mine network layout and ventilation arrangement chosen, and (3) the results of the calculated steady state pressure and airflow distribution. 


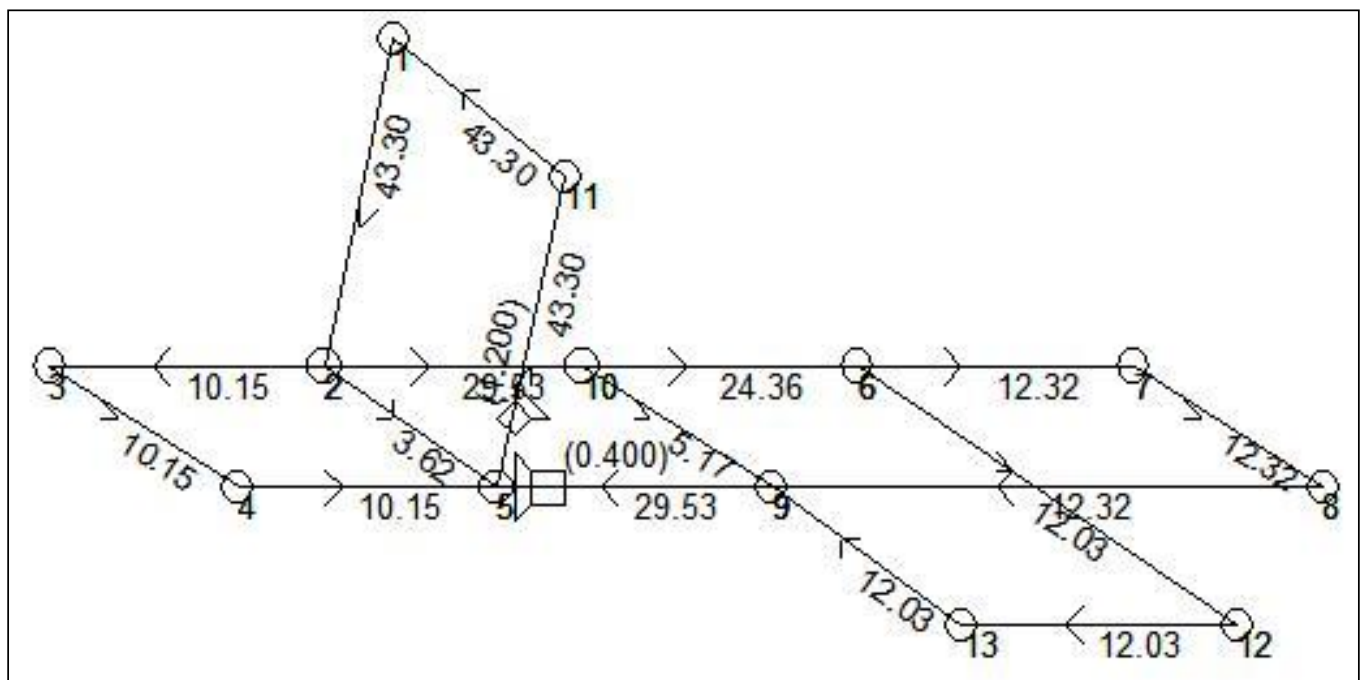

Figure 3: This schematic shows a simple small-scale mine ventilation network.

Source: Calizaya et al. (1987).

Figure 3 displays the node numbers at the ends of each airway and shows the direction and value of the calculated airflows $\left[\mathrm{m}^{3} / \mathrm{s}\right]$ on each branch. A main surface fan and a booster fan are placed within the branches flowing from node 5 to 11 and from node 9 to 5 , respectively. The layout of the mine network and location and duty of each component of the ventilation system are specified by the input data set prior to evaluating the steady state airflow and pressure distribution within the mine ventilation network. A comparative analysis of the results produced by the ventilation arrangements examined resolves the best solution in terms of air power costs.

\section{Primary Mine Ventilation Network Optimization Problem}

The PMVN optimization problem is defined by the objective function presented in Equation (1) coupled to the system of equations defined by Equations (2) to (14), detailed in the appendix. The objective is to minimize the fan power cost of a mine ventilation network, while delivering the airflow distribution required by the mine activities. Equations (13) and (14) represent the nonnegativity and nature of the variables, respectively. The PMVN optimization problem is 
nonlinear and nonconvex, and the solution seeks to minimize the total air power delivered by the fans in the network ( $\mathrm{Wu}$ and Topuz 1998). The product of the fan pressure $\left(F_{j}\right)$ and the volumetric flow $\left(Q_{j}\right)$ passing through the fan in the $j^{\text {th }}$ branch of the network determines the air power consumed by each fan, as Equation (11) presents. The sum of all the individual fan air powers determines the total air power consumed within a mine ventilation network. For branches that do not contain a fan, the fan pressure drop term is set to zero. The energy contributed by fans, as Equation (12) represents, serves to generate the required airflow and pressure distribution through the mine ventilation network.

The solution to the optimization problem identifies the number, location, and duty (or operational point, which is a pressure and airflow combination) of fans and regulators; these fans and regulators generate the airflow and pressure distribution to minimize the total fan air power or energy consumption of the mine, while delivering the airflow requirements at all the locations fixed by engineers.

Once the layout of a mine ventilation network is defined, a number of variants of the optimization problem may be solved. These variants may be categorized as (1) natural or free flow splitting, (2) controlled flow splitting, and (3) semi-controlled flow splitting. The general formulation of the initially defined PMVN corresponds to semi-controlled flow splitting. This variant has two types of subvariants, depending on the solution method, which we explain below in this section.

The optimization problem that considers the first variant, the natural or free flow splitting (FS), seeks to find the airflow and pressure distribution created by fans, the natural ventilation pressure, and the frictional and shock pressure losses within the airways. The natural ventilation pressure is formed by the difference in density caused by the different temperatures of the air 
flowing in the tunnels. In this variant, FS, Equation (16) is added to the PMVN to guarantee that the regulators are not used to control the flow distribution. The airflow in the network is split between the different branches according to their relative total resistance and the pressure distribution provided by the fans. In this case, the regulator pressure loss $R_{j}$ is set to zero (Acuña et al. 2010a).

The optimization problem that considers the second variant, the controlled flow splitting, is a different kind of problem. Because the airflows are known for all branches in the controlled flow splitting, the initial nonlinear optimization problem transforms into a linear optimization problem. The remaining decision variables are the fan pressures and the regulator resistances located in the mine ventilation network. The objective remains to minimize the fan air power, subject to delivering a minimum airflow quantity to all branches of the network. This variant is solved using linear programming methods.

The PMVN represents the third variant, the semi-controlled flow-splitting problem variant, which determines the installation of regulators and (or) booster fans within a predefined subset of the network branches, while the flows in the rest of the network branches are allowed to split naturally, to achieve the required airflow distribution at the lowest fan air power. For semi-controlled splitting (SC), there are two separate subvariants of the problem. Type 1, semicontrolled 1 (SC1) allows regulators only within the specified airflow branches; Equation (17) is added to the PMVN. Type 2, semi-controlled 2 (SC2) permits regulators in any set of permissible branches of the network. The SC2 variant corresponds to the PMVN.

To summarize, in the first variant, FS, the mine considers fans as the only means to regulate the airflow and pressure distribution. In the third variant, semi-controlled flow splitting, not only fans, but also regulators are considered for regulating the airflow and pressure 
distribution. These variants are the two most common cases found in the mining industry. The second variant, in which the airflow is known and can be regulated in all branches, is hard to find in the industry, but it can be used to find solutions to the semi-controlled flow-splitting variant, as we show in the next section.

Even if regulators are expected to dissipate energy in a mine ventilation network, Wang (1999) and Acuña et al. (2010a) conclude that adding regulators can reduce the overall energy consumed by the fans installed within semi-controlled flow networks. The addition of regulators can change fan duty points, reduce leakage, and create a more efficient airflow distribution.

\section{Solution Methods}

In recent years, several studies have explored the use of mathematical methods to minimize the power consumption of mine ventilation networks; these include Wu and Topuz (1989), Calizaya et al. (1987), Wang (1989), Barnes (1989), Jacques (1991), Huang and Wang (1993), Kumar et al. (1995), Wu and Topuz (1998), Lowndes and Yang (2004), Lowndes et al. (2005); Acuña et al. (2010a), and Acuña et al. (2010b). The mathematical approaches of these studies include operations research methods.

Wu and Topuz (1989) present a review of optimization methods that solve the controlled and semi-controlled flow-splitting variants of the optimization problem. All methods evaluate the minimum air power consumed to deliver a specified airflow distribution. A controlled flowsplitting problem exists when the airflow in all branches of a network is known.

In this review, the authors identify three classes of methods to solve the controlled flowsplitting variant of the optimization problem: (1) linear programming techniques, including the network simplex method, (2) critical path crashing techniques, including applications of the 
critical path method (CPM), and (3) a combination of CPM and cutset (i.e., a minimal collection of branches whose removal separates the network into two parts) operations. The CPM determines the longest path between two locations in the network. For mine ventilation networks, the longest path of connected branches corresponds to the path of greatest resistance (i.e., the path that makes the largest contribution to the total air power consumed by the ventilation network). Thus, the solution may consist of installing a booster fan within a highresistance branch to compensate for the high frictional pressure drop. The method then recalculates the new total air power consumed by the network. The process searches for solutions that minimize the value of the objective function based on air power consumption. However, the ventilation engineer needs to consider the practicality of all the solutions proposed. The third class of solution methods is a combination of CPM and cutset operation. A fundamental cutset is the minimum number of branches whose removal defines the tree of branches that connect all the nodes of the network. These branches characterize the mesh flow and pressure distribution within the network, allowing for the determination of location and calculation of the pressure value of a potential booster fan. Thus, this method first uses a CPM to identify the location and value of any additional regulator resistances and then employs cutset operations to determine the location and pressure duties of any additional booster fans required.

Two classes of methods were identified to solve the semi-controlled flow-splitting variant of the optimization problem. We describe the first method, developed by Calizaya et al. (1987), in this section. The second method consists of five iterative steps, which allow the evaluation of the air power consumed by the network, considering changes of the airflow quantities in each branch. The core of the method is a gradient technique. We present a small network consisting of six junctions and eight branches. 
Wu and Topuz (1989) remark that (1) none of the solution methods considers the total costs of a mine ventilation network, which include the investment costs associated with the purchase, installation, and maintenance of the fans and regulators, and (2) "further research work is therefore needed" (p. 360).

The optimization problem, which Calizaya et al. (1987), Kumar et al. (1995), and Lowndes et al. (2005) study, considers the type 1 semi-controlled flow (SC1) variant. All the proposed solution techniques use an iterative algorithm to determine the resistance values of the regulators needed in the network. These methods restrict regulators to a prespecified selection of branches, which may include airways leading to the working faces. The methods that Wang (1989), Barnes (1989), Jacques (1991), Huang and Wang (1993), and Wu and Topuz (1998) propose are applicable to the type 2 semi-controlled flow (SC2) variant of the optimization problem, in which a regulator may placed in any branch of the network. Acuña et al. (2010b) consider the free flow-splitting variant of the optimization problem, in which regulators are not permitted. Except for Wang (1989) and Acuña et al. (2010b), all the studies in Table 2 claim to achieve a local optimal solution for the variant of the PMVN optimization problem considered. However, none of these studies provides proof of global optimality.

Calizaya et al. (1987) consider the solution of the type 1 semi-controlled flowoptimization problem by employing a hybrid solution method based on the repeated application of a commercial steady state mine ventilation network program. These solutions produce a set of linear approximations to the branch resistance curves, gained from an evaluation of the pressure losses produced by the regulators installed in each of the specified airflow branches. They then determine the minimum fan power consumed from a graphical solution of the intersection of the 
linear approximations to the regulator resistance curves. The application of this technique requires the definition of an initial set of feasible solutions, against which the optimality of the generated solutions is compared.

Wang (1989) explores the use of a single-step Newton-Raphson iterative method to solve the type 2 semi-controlled flow variant of the optimization problem and apply it to a small ventilation network. The method described uses a set of multiple initial values that converges to a local optimum. The application of this solution method divides the problem into dependent and independent variables for the airflow and the pressure, and recasts the general formulation of the problem based on this smaller set of parameters. The solution method permits the installation of ventilation devices (i.e., fans or regulators) to achieve the desired airflow distribution.

Barnes (1989) decomposes the type 2 semi-controlled flow variant of the optimization problem into two problems: (1) the determination of the airflow distribution problem, and (2) the evaluation of the pressure distribution required to generate the computed airflow distribution. Barnes employs "a network adaptation of the classical nonlinear programming loop between a search for an improving direction, followed by a one dimensional optimization in the current improving direction" (p. 399). In simple terms, the method seeks an improvement solution search direction, followed by the calculation of the step in the current improvement direction. The algorithm starts with a possible set of flows and then incrementally improves the flows while preserving feasibility. The algorithm ends when no improvement direction is possible, as Barnes (1989) indicates.

Jacques (1991) presents a heuristic optimization approach to compute the operational duties for ventilation devices (e.g., fans and regulators), which satisfy the required airflow distribution, to solve the type 2 semi-controlled flow variant of the optimization problem. The 
solution method can quickly respond to the daily changes in the demands placed on a real mine ventilation system. The heuristic concentrates on minimizing the airflow deviation from the requirements instead of minimizing the air power, as in other approaches. The technique defines the airflows that minimize the deviation from the airflow requirements, and then uses Kirchhoff's second law to determine the pressure required from fans and regulators available. As a result, the heuristic achieves the required airflow distribution by iteratively changing the duty points of fans and regulators. However, the method cannot locate fans and (or) regulators. Jacques (1991) indicates "when automatic control is aimed at, a method such as the one described here should be integrated as a module into an expert system software package which is destined in the future to take over from the ventilation officer" (p. 415).

Huang and Wang (1993) propose an iterative approach to solve the type 2 semicontrolled flow variant of the optimization problem based on a generalized reduced gradient (GRG) method that employs multiple initial values. The objective function minimizes the total air power delivered by the installed fans. The authors provide a detailed description of the major computational steps involved in applying the GRG method, which are (1) the determination of a search direction, and (2) the calculation of the step in the improvement direction. "The relative error is used as the termination criterion" (p. 157), considering that "air quantity in a branch is usually within a hundred m3/s" (p. 157) and "fan or regulator pressure could be as high as a few thousand $\mathrm{Pa} "$ (p. 157). The presentation of the solution for a small-scale mine ventilation network model, consisting of 18 branches and nine nodes, illustrates the use of the method.

Kumar et al. (1995) present a two-step algorithm to solve the type 1 semi-controlled flow variant of the optimization problem. The first step determines the best duty and location of the main surface fan(s) using the CPM. The CPM determines the largest pressure drop of the 
network, which is used to calculate the pressure value of the fan(s). The second step identifies the pressure duty of the underground booster fan(s), for predefined locations, using a four-stage heuristic approach that includes a Fibonacci and cyclic search routines (Bazaraa and Shetty 1979), coupled with the use of a steady state mine ventilation network program. The second phase of the algorithm determines a search direction and a step in the improvement direction. The objective function minimizes the air power consumed by fans. The best solution identifies that only one of the branches with specified airflow does not need to have a regulator installed to achieve the required airflow distribution, which minimizes the energy consumed by regulators.

$\mathrm{Wu}$ and Topuz (1998) propose a solution algorithm to the type 2 semi-controlled flow variant of the optimization problem. The algorithm employs a combination of the special ordered sets construct, the branch-and-bound algorithm, and linearization techniques, as Beale and Forrest (1976), Bertsekas (2003), and Luenberger and Ye (2008), respectively, discuss. The proposed algorithm transforms the initial nonlinear, nonconvex programming problem into a linear problem by introducing a special ordered set of variables. Then, the branch-and-bound method is used to explore the special ordered set of variables by directly partitioning the problem into subproblems, repeating the same procedure for each subproblem. Each subproblem is a linear programming model, because at that stage, the airflows have been fixed and the resulting problem is that of controlled flow splitting, which is linear. The resulting solution determines the fan pressures and regulator resistances necessary to obtain the required airflow distribution.

Lowndes and Yang (2004) and Lowndes et al. (2005) develop and apply a genetic algorithm, coupled with a steady state mine ventilation network program, to solve the type 1 semi-controlled flow variant of the optimization problem. The resulting algorithm is tested with the primary mine ventilation system of the former Chilean El Indio mine. The genetic algorithm 
search is guided by a fitness function composed of the total consumed air power coupled to a penalty function employed to reject the solutions that do not deliver the necessary airflow at the specified airflow branches. The genetic algorithm discovers the location and pressure of underground booster fans needed to support a main fan in the delivery of the required airflow distribution. A commercial steady state mine ventilation network program determines the values of any regulator resistances installed in the specified airflow branches. The study concludes that the genetic algorithm identifies an optimal solution when 3 out of 16 underground booster fans are installed within the network. Acuña et al. (2010a) and Acuña et al. (2010b) extend the initial solution method proposed by Lowndes and Yang (2004) and Lowndes et al. (2005) to solve the free flow-splitting variant of the optimization problem, using a genetic algorithm coupled with a steady state mine ventilation network program.

The three solution methods, which Calizaya et al. (1987), Lowndes et al. (2005), and Acuña et al. (2010a) propose to solve the type 1 semi-controlled flow variant of the optimization problem, employ a repeated application of a commercial steady state mine ventilation network program to evaluate the airflow and pressure distribution and the operating air power costs; they couple this program with an optimization technique. The use of the steady state mine ventilation network program to evaluate each potential solution takes much of the computational time needed to find a solution for a large mine ventilation network. Lowndes et al. (2010) propose the use of metaheuristic models and artificial neural networks to replace the repeated application of the conventional mine ventilation network program; their results are encouraging in terms of the time required to find the solution of the type 1 semi-controlled flow variant of the optimization problem. 
Table 2 presents a summary of the PMVN optimization problem solution methods reported in a series of recent studies published in the literature. The studies are subclassified according to (1) the type of flow-splitting problem solved, (2) the form of the objective function used, (3) the degree of optimality claimed, and (4) the solution method used.

We note that as the computational capabilities of computers have increased, the sizes of the problems that may be considered have increased from 2 to 3 fan locations and, at most, 20 branches (see Table 2), to sizes such as at the El Indio mine, which has 16 fan locations and 242 branches. Mines can have up to 20 primary mine ventilation fan locations and branches that number between 500 and 3000 .

Only the studies presented by Lowndes et al. (2005) and Acuña et al. (2010b) include the computational times associated with each solution method; the other studies in Table 2 do not report it. We see a need to define an established set of representative benchmark mine ventilation networks against which the computational speed and efficiency of future solution methods may be compared.

\section{Conclusions}

The paper presents a review of the current state of the art of PMVN optimization methods. Currently, mine ventilation engineers, guided by good practice and experience, employ existing mine ventilation network programs to determine the best mine ventilation configuration, using an iterative solution method to deliver the required airflow distribution at minimum cost. This solution method is time consuming and may not always identify the best solution. The use of suitable optimization algorithms may improve the solutions identified and the time required to obtain them. Several algorithms developed to solve the mine ventilation network optimization problem have produced encouraging results. Most of the published studies reviewed claim to 
achieve locally optimal solutions; however, none provides mathematical proof of global optimality. The results obtained from the algorithms developed to solve the three classes of flowsplitting network problems are encouraging, but some important gaps in knowledge still exist. For most of the solution methods reported in the literature, the times required to compute them are not provided.

\section{Appendix: Primary Mine Ventilation Network (PMVN) Model}

Let

- $\boldsymbol{A}=$ the network incidence matrix that codes the branch interconnections, with $a_{i j}$ coefficients defined as follows:

$\circ \quad a_{i j}=1$ if the $j^{\text {th }}$ branch connects to the $i^{\text {th }}$ junction and the air flows out of the $i^{\text {th }}$ junction;

$\circ \quad a_{i j}=-1$ if the $j^{\text {th }}$ branch connects to the $i^{\text {th }}$ junction and the air flows into the $i^{\text {th }}$ junction;

$\circ \quad a_{i j}=0$ if the $j^{\text {th }}$ branch is not connected to the $i^{\text {th }}$ junction;

- $A_{j}=$ the average cross-sectional area of branch $j\left(\mathrm{~m}^{2}\right)$;

- $\quad \boldsymbol{B}=$ the fundamental mesh matrix of the ventilation network that identifies the branches connected within each mesh, with $b_{i j}$ coefficients defined as follows:

○ $\quad b_{i j}=1$ if the $j^{\text {th }}$ branch is contained in the $i^{\text {th }}$ mesh and the flow within this branch is in the same direction as that defined for the mesh;

$\circ b_{i j}=-1$ if the $j^{\text {th }}$ branch is contained in the $i^{\text {th }}$ mesh and the flow within this branch is in the opposite direction as that defined for the mesh;

○ $\quad b_{i j}=0$ if the $j^{\text {th }}$ branch is not contained in the $i^{\text {th }}$ mesh; 
- $B F=$ the set of braches where fans might be installed, $|B F| \leq N b$;

- $B R=$ the set of braches where regulators might be installed, $|B R| \leq N b$;

- $C=$ the energy cost (currency units per kilowatt hour, $\$ \mathrm{USD} / \mathrm{kWh}$ );

- $\quad H R=$ the hours the fans will be running (h);

- $\quad k_{j}=$ the friction factor of branch $j\left(\mathrm{~kg} / \mathrm{m}^{3}\right)$;

- $\quad l_{j}=$ the lower bound for airflow of branch $j\left(\mathrm{~m}^{3} / \mathrm{s}\right)$;

- $\quad N b=$ the number of branches in the ventilation network;

- $N m=$ the number of fundamental meshes in the ventilation network, $N m=N b-N n+1$;

- $\quad N n=$ the number of nodes or junctions in the ventilation network;

- $\quad M_{i}=$ the fundamental mesh $i$ (closed path) in the ventilation network;

- $\quad N_{j}=$ the natural ventilation pressure drop of branch $j\left(\mathrm{kPa}\right.$, i.e., $\left.\mathrm{kN} / \mathrm{m}^{2}\right)$;

- $\quad p_{j}=$ the perimeter of branch $j(\mathrm{~m})$;

- $\quad r_{j}=$ the total resistance of branch $j\left(\mathrm{Ns}^{2} / \mathrm{m}^{8}\right)$;

- $S A B=$ the set of specified airflow branches, $|S A B| \leq N b$;

- $\quad s_{j}=$ the length of branch $j(\mathrm{~m})$;

- $\quad s_{j}^{\text {equiv }}=$ the shock loss equivalent length of branch $j(\mathrm{~m})$;

- $u_{j}=$ the upper bound for the airflow of branch $j\left(\mathrm{~m}^{3} / \mathrm{s}\right)$;

- $\rho_{j}=$ the density of the air of branch $j\left(\mathrm{~kg} / \mathrm{m}^{3}\right)$;

- $\quad \eta_{j}=$ the efficiency of the fan installed in $\operatorname{branch} j$ (as a fraction between 0 and 1 ).

The decision variables are defined as:

- $\quad F_{j}=$ the pressure developed by fan installed in branch $j(\mathrm{kPa})$; 
- $Q_{j}=$ the airflow quantity flowing through branch $j\left(\mathrm{~m}^{3} / \mathrm{s}\right)$;

- $s_{j}^{r e g}=$ the shock-loss-equivalent length of the regulator in branch $j(\mathrm{~m})$.

The auxiliary variables are defined as:

- $\quad E_{j}=$ the energy cost of the fan air power contribution into branch $j$ (\$USD);

- $\quad H_{j}=$ the pressure drop of branch $j(\mathrm{kPa})$;

- $\quad L_{j}=$ the frictional pressure drop of branch $j(\mathrm{kPa})$;

- $\quad P_{j}=$ the fan air power contribution into branch $j(\mathrm{~kW}$, i.e., $\mathrm{kNm} / \mathrm{s})$;

- $r_{j}^{*}=$ the total shock-loss-equivalent resistance of the regulator in branch $j\left(\mathrm{Ns}^{2} / \mathrm{m}^{8}\right)$;

- $\quad R_{j}=$ the regulator pressure drop of branch $j(\mathrm{kPa})$;

- $Z=$ the sum of fans air power of the mine $(\mathrm{kW})$.

\section{PMVN Model (PMVNM)}

$(\mathrm{PMVNM}) \quad \min \sum_{j \in B F} \frac{F_{j} Q_{j}}{\eta_{j}}=Z$,

subject to

$\sum_{j=1}^{N b} a_{i j} Q_{j}=0 \quad \forall i \in\{1, \ldots .,(n-1)\}$

$L_{j}=r_{j} Q_{j}^{2} \quad \forall j \in\{1, \ldots ., b\}$

$r_{j}=\left(\frac{k_{j} p_{j}\left(s_{j}+s_{j}^{\text {equiv }}\right)}{A_{j}^{3}}\right) \quad \forall j \in\{1, \ldots, b\}$

$R_{j}=r_{j}^{*} Q_{j}^{2} \quad \forall j \in B R$ 
$r_{j}^{*}=\left(\frac{k_{j} p_{j} s_{j}^{r e g}}{A_{j}^{3}}\right) \quad \forall j \in B R$

$H_{j}=L_{j}+R_{j}-F_{j}-N_{j} \quad \forall j \in\{1, \ldots ., b\}$

$\sum_{j=1}^{N b} b_{i j} H_{j}=0 \quad \forall i \in\{1, \ldots ., m\}$

$l_{j} \leq Q_{j} \quad \forall j \in\{1, \ldots, b\}$

$Q_{j} \leq u_{j} \quad \forall j \in\{1, \ldots ., b\}$

$P_{j}=\frac{F_{j} Q_{j}}{\eta_{j}} \quad \forall j \in\{1, \ldots ., b\}$

$E_{j}=\frac{F_{j} Q_{j}}{\eta_{j}} \cdot H R \cdot C \quad \forall j \in\{1, \ldots, b\}$

$F_{j}, Q_{j}, E_{j}, H_{j}, L_{j}, P_{j}, R_{j}, s_{j}^{r e g}, r_{j}^{*} \geq 0 \quad \forall j \in\{1, \ldots ., b\}$

$F_{j}, Q_{j}, E_{j}, H_{j}, L_{j}, P_{j}, R_{j}, s_{j}^{r e g}, r_{j}^{*} \in \mathbb{R} \quad \forall j \in\{1, \ldots ., b\}$

\section{Other relevant equations}

$$
\begin{array}{ll}
\sum_{j=1}^{N b} a_{i j} \rho_{j} Q_{j}=0 & \forall i \in\{1, \ldots .(n-1)\} \\
s_{j}^{r e g} \leq 0 & \forall j \in\{1, \ldots ., b\} \\
s_{j}^{r e g} \leq 0 & \forall j \in(B R \cap S A B)^{c}
\end{array}
$$




\section{References}

Acuña E, Hall S, Lowndes I (2010a) Free and semi controlled splitting network optimisation using GAs to justify the use of regulators. Castro R, Emery X, Kuyvenhoven R, eds. Proc. IV Internat. Conf. Mining Innovation (Gecamin, Santiago, CL), 79-87.

Acuña E, Maynard R, Hall S, Hardcastle SG, Li G, Lowndes IS, Tonnos A (2010b) Practical mine ventilation optimization based on genetic algorithms for free splitting networks. Proc. 13th Mine Ventilation Sympos. (Society for Mining, Metallurgy \& Exploration, Englewood, CO), 379-385.

Barnes RJ (1989) A partial solution to optimal mine ventilation network design. Proc. 4th US Mine Ventilation Sympos. (Society for Mining, Metallurgy \& Exploration, Englewood, CO), 395-404.

Bazaraa MS, Shetty CM (1979) Nonlinear Programming: Theory and Algorithms (John Wiley \& Sons, New York), 252-330.

Beale EML, Forrest JJH (1976) Global optimization using special ordered sets. Math. Programming.10(1):52-69.

Bertsekas D (2003) Nonlinear Programming (Athena Scientific, Belmont, MA).

Calizaya F, McPherson MJ, 'Mousset-Jones P (1987) An algorithm for selecting the optimum combination of main and booster fans in underground mines. Proc. 3rd US Mine Ventilation Sympos. (Society for Mining, Metallurgy \& Exploration, Englewood, CO), $408-417$.

Cross H (1936) Analysis of flow in networks of conduits or conductors. Univ. Illinois Bull. $\operatorname{XXXIV(22):1-32.~}$ 
Hardcastle SG (1995) 3D-Canvent: An interactive mine ventilation simulator. Proc. 7th US Mine Ventilation Sympos. (Society for Mining, Metallurgy \& Exploration, Englewood, CO), $467-472$.

Huang C, Wang YJ (1993) Mine ventilation network optimization using the generalized reduced gradient method. Proc. 6th US Mine Ventilation Sympos. (Society for Mining, Metallurgy \& Exploration, Englewood, CO), 153-161.

Jacques EJ (1991) A solution to the optimal setting of air flow-control devices in a ventilation network. Proc. 5th US Mine Ventilation Sympos. (Society for Mining, Metallurgy \& Exploration, Englewood, CO), 411-415.

Kumar GV, Sastry VR, Krishna Rao GV (1995) Minimizing power consumption in multiple fan networks by optimum fan selection. Proc. 7th US Mine Ventilation Sympos. (Society for Mining, Metallurgy \& Exploration, Englewood, CO), 491-497.

Lowndes IS, Tuck MA (1995) Review of mine ventilation system optimization. Trans. Institution Mining Metallurgy, Section A 105(a):A114-A126.

Lowndes IS, Yang ZY (2004) The application of GA optimisation method to the design of practical ventilation systems for multi-level metal mine operations. Mining Tech. 113(1):43-58.

Lowndes IS, Fogarty T, Yang ZY (2005) The application of genetic algorithms to optimize the performance of a mine ventilation network: the influence of coding method and population size. Soft Comput. 9(7):493-506.

Lowndes IS, Dandy GC, Marshall TS, Schmidt TB, Simpson NG, Raynor GP (2010) Optimization of mine ventilation networks using genetic algorithms and artificial neural networks. Hardcastle SG, McKinnon DL, eds. Proc. $13^{\text {th }}$ US/North Amer. Mine 
Ventilation Sympos. (Society for Mining, Metallurgy \& Exploration, Englewood, CO), 441-447.

Luenberger DG, Ye Y (2008) Linear and Nonlinear Programming (Springer, New York).

Marx W, Belle BK (2002) Simulating airflow conditions in a South African coal mine, using the VUMA-network simulation software. Accessed October 1, 2013, http://www.vuma.co.za/pdf/VumaC.pdf.

Moll ATJ, Lowndes IS (1992) The application of graph theory to mine ventilation networks Bull. Inst. Math. Appl. 28(6-8):103-106.

McPherson MJ (1993) Subsurface Ventilation and Environmental Engineering (Chapman \& Hall, London).

Wallace Jr. KG (2001) General operational characteristics and industry practices of mine $\begin{array}{llll}\text { ventilation } & \text { systems. } & \text { Accessed } & \text { October }\end{array}$ http://www.mvsengineering.com/files/Publications/07th_INT-1.pdf.

Wang YJ (1989) A procedure for solving a more generalized system of mine ventilation network equations. Proc. 4th US Mine Ventilation Sympos. (Society for Mining, Metallurgy \& Exploration, Englewood, CO), 419-424.

Wang YJ (1999) Minimizing air power in a ventilation network using regulators in nonfixed branches. J. Mine Ventilation Soc. 52(2):39-43.

Widzyk-Capehart E, Watson B (2001) Agnew gold mine expansion, mine ventilation expansion evaluation using Ventsim. Accessed October 1, 2013, http://www.ventsim.com/files/Agnew\%20Gold\%20Mine\%20Expansion.pdf. 
Wu X, Topuz E (1989) Comparison of methods for determination of booster fan locations in underground mines. Proc. 4th US Mine Ventilation Sympos. (Society for Mining, Metallurgy \& Exploration, Englewood, CO), 355-362.

Wu XS, Topuz E (1998) Analysis of mine ventilation systems using operations research methods. Internat. Trans. Oper. Res. 5(4):245-254. 